\title{
Experiences and attitudes of medical professionals on treatment of end-of-life patients in intensive care units in the Republic of Croatia: a cross-sectional study
}

Diana Špoljar ${ }^{*}$, Marinko Vučić ${ }^{2}$, Jasminka Peršec ${ }^{1}$, Vlasta Merc $^{1}$, Tatjana Kereš ${ }^{1}$, Radovan Radonić $^{3}$, Zdravka Poljaković ${ }^{3}$, Višnja Nesek Adam ${ }^{4}$, Nenad Karanović ${ }^{5}$, Krešimir Čaljkušić ${ }^{5}$, Željko Župan ${ }^{6}$, Igor Grubješić ${ }^{6}$,

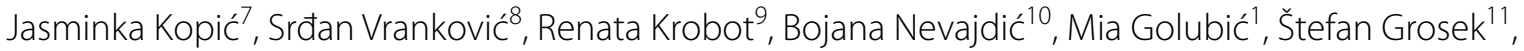
Mirjana Kujundžić Tiljakk ${ }^{12}$, Andrija Štajduhar ${ }^{12}$, Dinko Tonković ${ }^{3}$ and Ana Borovečki ${ }^{12}$

\begin{abstract}
Background: Decisions about limitations of life sustaining treatments (LST) are made for end-of-life patients in intensive care units (ICUs). The aim of this research was to explore the professional and ethical attitudes and experiences of medical professionals on treatment of end-of-life patients in ICUs in the Republic of Croatia.

Methods: A cross-sectional study was conducted among physicians and nurses working in surgical, medical, neurological, and multidisciplinary ICUs in the total of 9 hospitals throughout Croatia using a questionnaire with closed and open type questions. Exploratory factor analysis was conducted to reduce data to a smaller set of summary variables. Mann-Whitney $U$ test was used to analyse the differences between two groups and Kruskal-Wallis tests were used to analyse the differences between more than two groups.
\end{abstract}

Results: Less than third of participants (29.2\%) stated they were included in the decision-making process, and physicians are much more included than nurses $(p<0.001)$. Sixty two percent of participants stated that the decisionmaking process took place between physicians. Eighteen percent of participants stated that 'do-not-attempt cardiopulmonary resuscitations' orders were frequently made in their ICUs. A decision to withdraw inotropes and antibiotics was frequently made as stated by $22.4 \%$ and $19.9 \%$ of participants, respectively. Withholding/withdrawing of LST were ethically acceptable to $64.2 \%$ of participants. Thirty seven percent of participants thought there was a significant difference between withholding and withdrawing LST from an ethical standpoint. Seventy-nine percent of participants stated that a verbal or written decision made by a capable patient should be respected. Physicians were more inclined to respect patient's wishes then nurses with high school education $(p=0.038)$. Nurses were more included in the decision-making process in neurological than in surgical, medical, or multidisciplinary ICUs $(p<0.001, p=0.005$, $p=0.023$ respectively). Male participants in comparison to female $(p=0.002)$, and physicians in comparison to nurses with high school and college education $(p<0.001)$ displayed more liberal attitudes about LST limitation.

*Correspondence: dianaspoljar@gmail.com

1 University Hospital Dubrava, Av. Gojka Šuška 6, 10000 Zagreb, Croatia

Full list of author information is available at the end of the article

(c) The Author(s) 2022. Open Access This article is licensed under a Creative Commons Attribution 4.0 International License, which permits use, sharing, adaptation, distribution and reproduction in any medium or format, as long as you give appropriate credit to the original author(s) and the source, provide a link to the Creative Commons licence, and indicate if changes were made. The images or other third party material in this article are included in the article's Creative Commons licence, unless indicated otherwise in a credit line to the material. If material is not included in the article's Creative Commons licence and your intended use is not permitted by statutory regulation or exceeds the permitted use, you will need to obtain permission directly from the copyright holder. To view a copy of this licence, visit http://creativecommons.org/licenses/by/4.0/. The Creative Commons Public Domain Dedication waiver (http://creativeco mmons.org/publicdomain/zero/1.0/) applies to the data made available in this article, unless otherwise stated in a credit line to the data. 
Conclusions: DNACPR orders are not commonly made in Croatian ICUs, even though limitations of LST were found ethically acceptable by most of the participants. Attitudes of paternalistic and conservative nature were expected considering Croatia's geographical location in Southern Europe.

Keywords: Intensive care units, End-of-life care, End-of-life decision-making, Ethics

\section{Background}

A certain percentage of patients in the intensive care units (ICUs) are at the ends of their lives and decisions about further diagnostic and treatment procedures are made accordingly. End-of-life decision-making is a process which involves physicians, nurses, patients and their families, and the goal is to decide whether to limit further (and which) treatments [1]. Both physicians and nurses find that most ethical dilemmas arise in their clinical practice relating to this subject $[1,2]$.

Studies have shown that withholding and withdrawing of treatment and shortening of the dying process were used less frequently in the southern European countries compared to the central or northern countries [3, 4]. It has also been shown that Catholic physicians and medical professionals are less inclined to follow a competent patient's wish to refuse a treatment that might be lifesaving $[5,6]$.

Ethicus-2, a more recent prospective, multinational, observational study shows that the limitation of life-sustaining treatment (LST) occurs in about $12 \%$ of patients admitted to ICUs. This study confirms that treatment limitations are much more common in North America, Australia/New Zealand and Northern Europe than in Africa, Latin America and Southern Europe, and withholding LST is more common than withdrawing [7].

Many countries have specific guidelines which offer support and assistance to medical professionals in the decision-making process [8-14]. Many guidelines underpin the notion of a team of medical professionals making such decisions, and nurses as parts of that team, as they often have an intimate insight into patients' lives, are acquainted with their wishes and provide emotional support [15-18]. Physicians from northern European regions are of the opinion that nurses are more involved in the decision-making process than physicians from central and southern regions [19]. However, nurses feel they are not included in the decision-making process nor that their opinion is valued [18-22].

Croatian law bans euthanasia and physician-assisted suicide, while advance directives are not legally binding. Furthermore, according to laws on health care and patients' rights, patients do not have the right to refuse treatment in case of mortal danger [23, 24]. There are no clearly defined national guidelines on end-of-life treatment and decision-making in Croatia. So far, an extensive, national survey on treatment of end-of-life patients has never been conducted in the Republic of Croatia, nor was Croatia ever included in a multinational survey of the type.

The aim of this research was to explore the professional and ethical attitudes and experiences of medical professionals on treatment of end-of-life patients in ICUs in the Republic of Croatia.

\section{Methods}

A cross-sectional study was conducted using a questionnaire among physicians and nurses working in surgical, medical, neurological, and multidisciplinary ICUs in the total of 9 hospitals throughout Croatia, including 4 clinical centres, 2 clinical hospitals and 3 general hospitals. General hospitals in Croatia provide treatment for basic and simpler medical conditions and are less equipped than clinical hospitals, which are associated with a university and provide treatment for more complicated conditions. A clinical centre is the medical institution of the highest level.

The study was aimed at all nurses and medical doctors-specialists who work full time or perform overnight shifts in the ICU. Not all medical doctors working in the ICU are specialists in critical care. Residents and physicians who are temporarily working in selected ICUs were excluded.

The questionnaires were handed to the ICU directors who informed the staff about the aim and the conduction of the research. A quiet place was provided for all participants to fill out the questionnaires, which were then collected by the directors in a way which ensured participants' anonymity and returned to the researcher. The ICU directors provided the total number of physicians and nurses working in the ICU to calculate the response rate.

The questionnaire was initially constructed by Groselj et al. for a cross-sectional, nation-wide study of experiences of Slovene ICU-physicians [25]. As Croatia and Slovenia are neighbouring countries that were once a part of the same federal republic and are now in a similar socio-economic situation, we opted for a questionnaire used there to make the comparisons easier.

The translations were conducted by registered translators and a back-translation was undertaken, meaning it was translated from Slovenian to Croatian, and back to 
Slovenian by another independent registered translator. The original Slovenian version and the back-translated Slovenian version were compared to check for quality and accuracy. It was comprehensively reviewed for linguistic, grammatical, and technical accuracy. Slight changes were made regarding the order of the questions, several questions were added, and the questionnaire was then validated for Croatian population.

The questionnaire consists of 4 parts with closed and open type questions (Additional File 1). The first part relates to general and demographic data, the second part explores the experiences of medical professionals regarding end-of-life decision-making and implementation of made decisions, while the third part explores the attitudes on the subject. The fourth part was intended for physicians only, as it consists of a made-up clinical scenario about a patient with a brain haemorrhage. The questionnaire was anonymous and took on average 15 min to complete.

A pilot study was conducted in a convenient sample of nurses and physicians in 2 different hospitals. Ethical clearance was obtained from the Ethics committee of the University of Zagreb-Medical school and from each participating hospital. The distribution and collection of the questionnaires took place from October 2018 to December 2019.

\section{Data analysis}

The data from the questionnaires were compiled into an Excel sheet and all data were analysed using Python programming language. Descriptive statistics were conducted on all data. Information gathered in the open type questions were scarce and therefore excluded from further analysis. Cronbach's alpha was used to measure internal consistency, and a coefficient of 0.70 or higher was considered acceptable. Exploratory factor analysis was conducted to reduce data to a smaller set of summary variables, and an oblique rotation (Promax) was used. Mann-Whitney U test was used to analyse the differences between two groups and Kruskal-Wallis tests were used to analyse the differences between more than two groups. Post-hoc analysis was conducted using the Holm-Bonferroni correction. Differences in categorical values were analysed with Yates's chi-squared test. The significance level was set at $p \leq 0.05$.

\section{Results}

\section{Pilot study}

The pilot study was conducted in a convenient sample of nurses and physicians in 2 different hospitals including 2 medical, 2 surgical and 2 neurological ICUs. The total response rate of the pilot study was $52.1 \%$, the total number of participants was 208; $72.1 \%$ were female,
$30.8 \%$ were physicians. Sixty-two and a half percent of physicians were anaesthesiologists, $23.4 \%$ were internal medicine physicians and $14.1 \%$ were neurologists. Since the questionnaire was not modified after the completion of the pilot study, the results from the pilot study were added to the results of the main study conducted in other hospitals.

\section{Characteristics of main study participants}

The study was conducted in 18 ICUs in 9 different hospitals, including 3 medical, 5 surgical, 6 neurological and 4 multidisciplinary ICUs. The total response rate of all included participants was $51.5 \%$, while physicians' response rate was $63.1 \%$ and nurses' $47.5 \%$.

Total number of participants was 438; $75.8 \%$ were female, $31.3 \%$ were physicians. Seventy percent of physicians were anaesthesiologists, $13.1 \%$ were internal medicine physicians and $16.8 \%$ were neurologists. Participants' mean age was 37.7 years $(\mathrm{SD} \pm 11.5)$ with work experience on average 15.3 years $(\mathrm{SD} \pm 108)$.

The other characteristics of study participants are listed in Table 1.

\section{Experiences of medical professionals regarding end-of-life decision-making and implementation}

Less than third of participants (29.2\%) stated they were included in the decision-making process. Physicians are much more included than nurses $(p<0.001)$, and participants younger than 31 years and with total work experience less than 10 years are less included than their older and longer working colleagues ( $p<0.001$ in both cases). Sixty two percent of participants stated that the decisionmaking process took place between physicians, and only $23.4 \%$ of participants stated that nurses were involved in the decision-making. Two thirds of participants (66.7\%) agreed that physicians were the ones who initiated the conversation about LST limitation, and only $2.5 \%$ said that nurses initiated such conversations.

Sixty percent of participants stated that verbal 'donot-attempt cardiopulmonary resuscitation' (DNACPR) orders were given, and $59.1 \%$ state that verbal orders were given for other types of LST limitations in their ICUs. A DNACPR order was always respected by $67.4 \%$ of participants, with male participants respecting such orders more than female $(p=0.042)$.

When asked about the frequency of limitation of LST in their ICU, $18 \%$ of participants stated that DNACPR orders were frequently made in their ICUs, in contrast to 49.5\% who stated that such decisions were rarely made; $13.7 \%$ of participants stated that therapy was frequently withheld, while $48.6 \%$ participants stated that such decisions were rarely made. A decision to withdraw inotropes and antibiotics was frequently made as stated by 
Table 1 Characteristics of study participants

\begin{tabular}{|c|c|c|c|c|c|}
\hline & $\begin{array}{l}\text { All } \\
\mathrm{N}(\%)\end{array}$ & $\begin{array}{l}\text { Physicians } \\
\mathrm{N}(\%)\end{array}$ & $\begin{array}{l}\text { Nurses } \\
\mathrm{N}(\%)\end{array}$ & $\begin{array}{l}\text { Male } \\
\mathrm{N}(\%)\end{array}$ & $\begin{array}{l}\text { Female } \\
\mathrm{N}(\%)\end{array}$ \\
\hline \multicolumn{6}{|l|}{ Vocation_education level } \\
\hline Physician—specialist & $137(31.3)$ & - & - & $60(59.4)$ & $77(23.2)$ \\
\hline Nurse-high school graduate & $159(36.3)$ & - & - & $23(22.8)$ & $134(40.4)$ \\
\hline Nurse_college graduate & $114(26.0)$ & - & - & $15(14.9)$ & $96(28.9)$ \\
\hline Nurse_-university graduate & $28(6.4)$ & - & - & $3(3.0)$ & $25(7.5)$ \\
\hline \multicolumn{6}{|l|}{ ICU type } \\
\hline Surgical & $219(50.0)$ & $66(48.2)$ & $153(50.8)$ & $56(55.5)$ & $161(48.5)$ \\
\hline Internal medicine & $54(12.3)$ & $18(13.1)$ & $36(12.0)$ & $13(12.9)$ & $40(12.1)$ \\
\hline Neurological & $75(17.1)$ & $23(16.8)$ & $52(17.3)$ & $13(12.9)$ & $62(18.7)$ \\
\hline Multidisciplinary & $90(20.6)$ & $30(21.9)$ & $60(19.9)$ & $19(18.8)$ & $69(20.8)$ \\
\hline \multicolumn{6}{|l|}{ Work in ICU } \\
\hline Every day & $330(75.3)$ & $61(44.6)$ & $269(89.4)$ & $69(68.3)$ & $256(77.1)$ \\
\hline Occasional & $84(19.2)$ & $75(54.7)$ & $9(3.0)$ & $31(30.7)$ & $53(16.0)$ \\
\hline Did not answer & $24(5.5)$ & $1(0.7)$ & $23(7.6)$ & $1(1.0)$ & $23(6.9)$ \\
\hline \multicolumn{6}{|l|}{ Hospital type } \\
\hline Clinical & $384(87.7)$ & $117(85.4)$ & $267(88.7)$ & $91(90.1)$ & $289(87.1)$ \\
\hline General & $54(12.3)$ & 20 (14.6) & 34 (11.3) & $10(9.9)$ & $43(13.0)$ \\
\hline
\end{tabular}

$22.4 \%$ and $19.9 \%$, respectively. Withdrawal of mechanic ventilation was never performed as stated by $55.5 \%$, the endotracheal tube was never removed as stated by $61.0 \%$, and hydration was never stopped as stated by $69.0 \%$ of participants.

Half of the participants $(49.1 \%)$ stated that family members/legal guardians were mostly or always included in the decision-making process. Detailed list of responses is shown in Table 2.

\section{Attitudes of medical professionals regarding end-of-life decision-making and implementation}

DNACPR orders and withholding/withdrawing of LST were ethically acceptable to $71.9 \%$ and $64.2 \%$ of participants, respectively. Thirty seven percent of participants stated they thought there was a significant difference between withholding and withdrawing LST from an ethical standpoint, with more participants working in general than in clinical hospitals $(p=0.020)$ having that opinion.

If the patient was incapacitated, $28.3 \%$ of participants stated that a team of physicians should decide about LST limitation, and $46.6 \%$ stated that such a decision should be made by a physician and the patient's family/legal guardians.

Most of the participants (79.5\%) stated that a verbal or written decision made by a capable patient should be respected. However, $55.2 \%$ of participants stated that they rarely or very rarely knew the patient's wishes regarding LST limitation.
When asked about which aspects of the decisionmaking process should be respected, $80.8 \%$ of participants stated that good medical practice, $79 \%$ stated that patient's interest, and 66\% stated that patient's autonomy should be respected.

Fifty eight percent of participants stated that family's wishes, $50.2 \%$ stated that religious principles, and $68.3 \%$ stated that legal regulations should be respected. Seventy six percent of participants stated that advanced directives (AD) should also be respected, however $67.1 \%$ of participants have never encountered an $\mathrm{AD}$ in their practice, and only one participant (0.2\%) stated they have encountered it often. Thirty eight percent and $13.5 \%$ of participants stated that treatment expenses and the need for ICU beds should be respected, respectively. Detailed list of responses is shown in Tables 3 and 4 .

\section{Exploratory factor analysis}

In order to reduce data to a smaller set of summary variables Exploratory factor analysis was conducted. We divided the data into two subsets: the first included the Likert type questions where the maximum value was 5 ( $1=$ strongly disagree $-5=$ strongly agree), and the second subset included questions where the maximum value was 3 or 4 . Barlett's test of sphericity was significant $(p<0.001)$ for both subsets of data. The Kaiser-MeyerOlkin measure of sample adequacy was 0.7330 for the first and 0.6962 for the second subset of data, indicating that the sampling is adequate for factor analysis, however middling. 


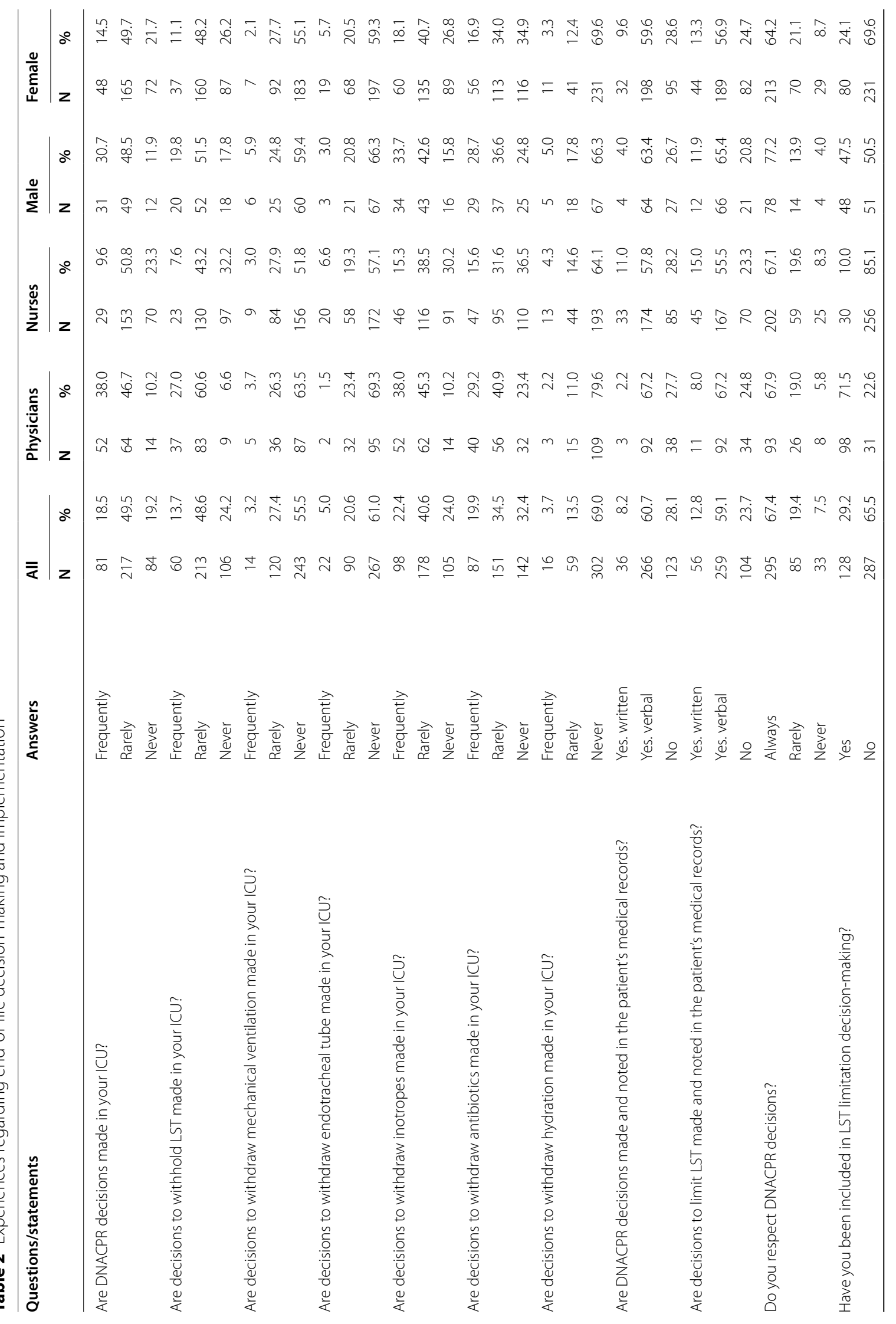




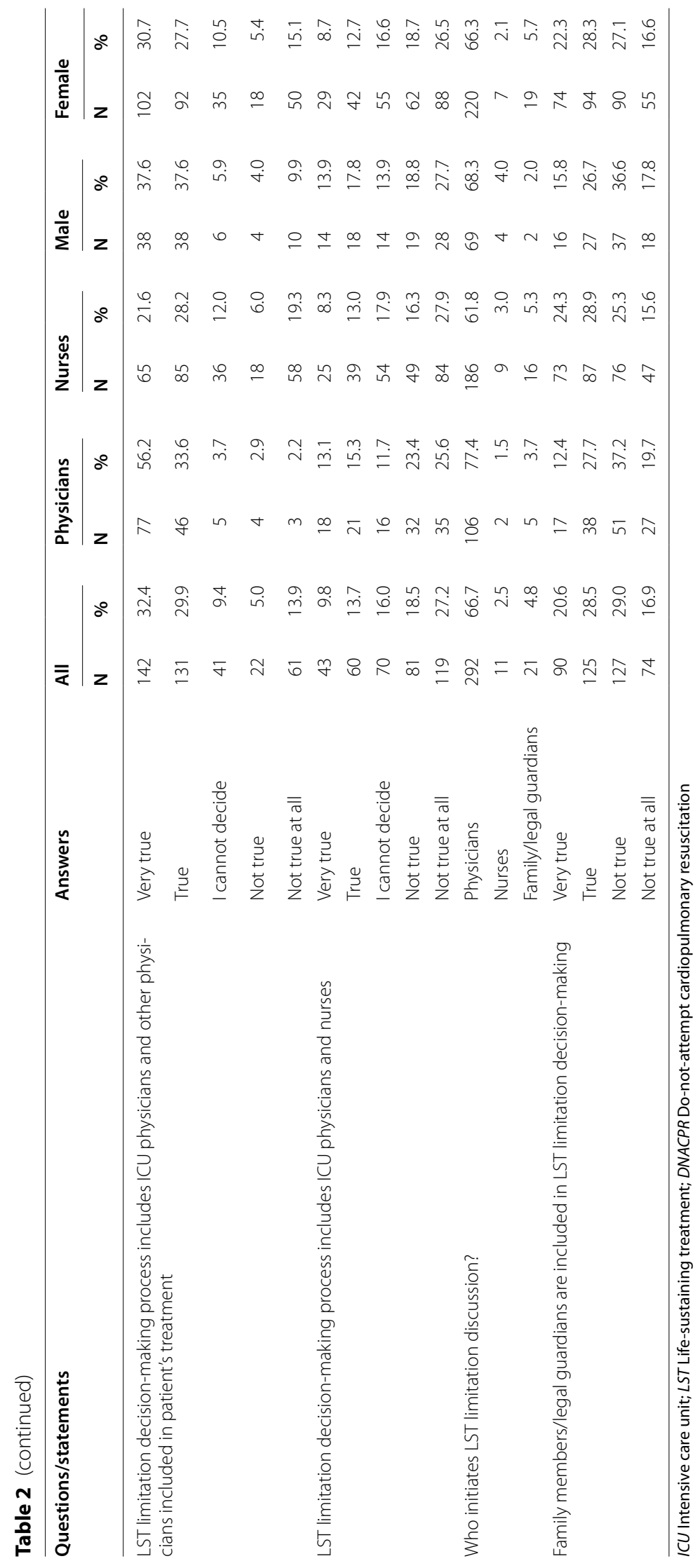




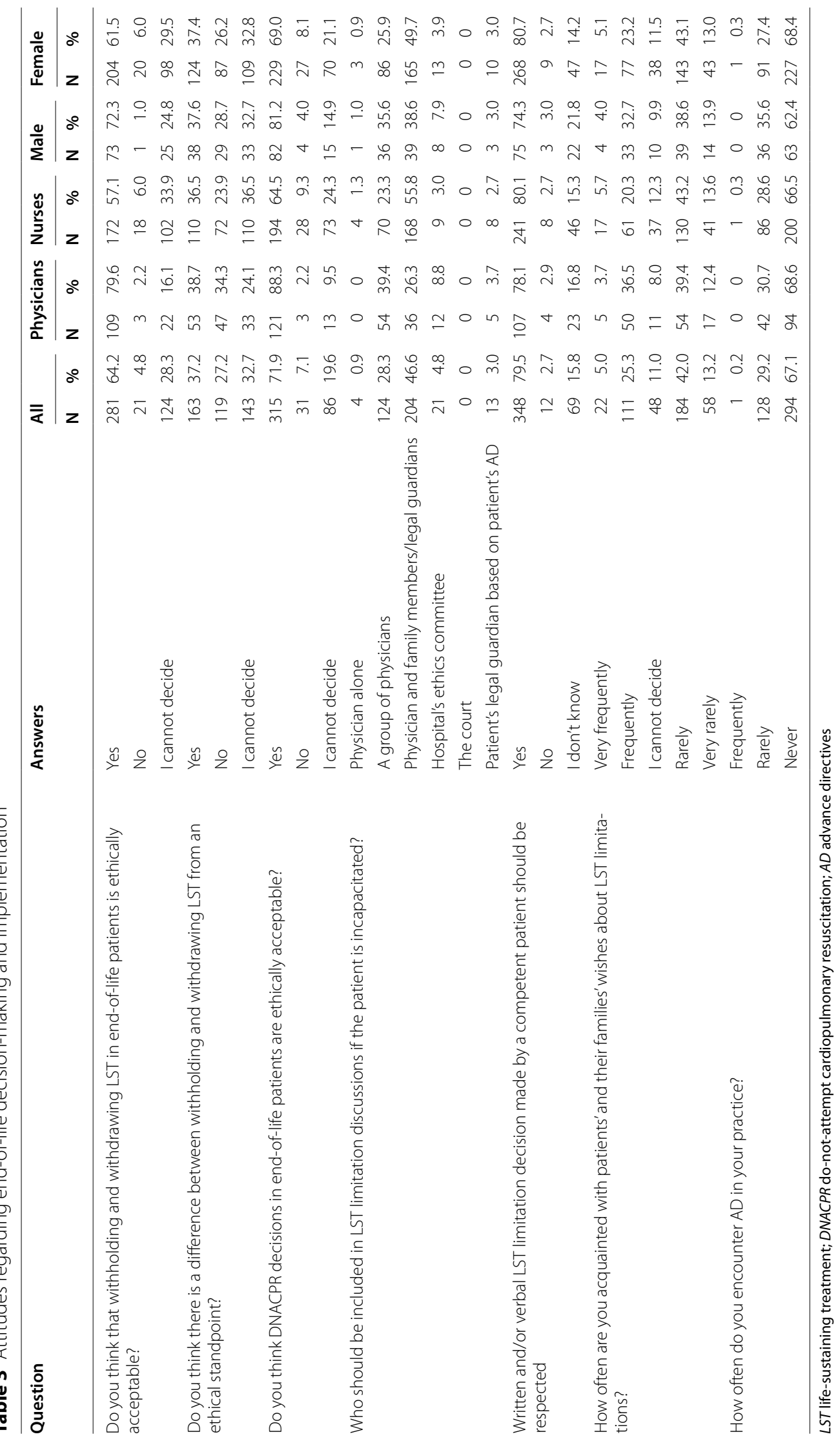


Table 4 Attitudes regarding which aspects should be respected in LST limitation decision-making

\begin{tabular}{|c|c|c|c|c|c|c|c|c|c|c|c|}
\hline \multirow{2}{*}{\multicolumn{2}{|c|}{$\begin{array}{l}\text { The following aspects should be respected in LST } \\
\text { limitation decision-making }\end{array}$}} & \multicolumn{2}{|l|}{ All } & \multicolumn{2}{|c|}{ Physician } & \multicolumn{2}{|c|}{ Nurses } & \multicolumn{2}{|c|}{ Male } & \multicolumn{2}{|c|}{ Female } \\
\hline & & $\mathbf{N}$ & $\%$ & $\mathbf{N}$ & $\%$ & $\mathbf{N}$ & $\%$ & $\mathbf{N}$ & $\%$ & $\mathbf{N}$ & $\%$ \\
\hline \multirow[t]{5}{*}{ Good medical practice } & I strongly agree & 241 & 55.0 & 89 & 65.0 & 152 & 50.5 & 59 & 58.4 & 179 & 53.9 \\
\hline & I agree & 113 & 25.8 & 31 & 22.6 & 82 & 27.2 & 29 & 28.7 & 84 & 25.3 \\
\hline & I cannot decide & 39 & 8.9 & 9 & 6.6 & 30 & 10.0 & 9 & 8.9 & 29 & 8.7 \\
\hline & I disagree & 9 & 2.1 & 1 & 0.7 & 8 & 2.7 & 1 & 1.0 & 8 & 2.4 \\
\hline & I strongly disagree & 12 & 2.7 & 1 & 0.7 & 11 & 3.7 & 1 & 1.0 & 11 & 3.3 \\
\hline \multirow[t]{5}{*}{ Patient's interests } & I strongly agree & 225 & 51.4 & 96 & 70.1 & 129 & 42.9 & 55 & 54.5 & 167 & 50.3 \\
\hline & I agree & 121 & 27.6 & 27 & 19.7 & 94 & 31.2 & 26 & 25.7 & 94 & 28.3 \\
\hline & I cannot decide & 51 & 11.6 & 8 & 5.8 & 43 & 14.3 & 17 & 16.8 & 34 & 10.2 \\
\hline & I disagree & 6 & 1.4 & 1 & 0.7 & 5 & 1.7 & 0 & 0 & 6 & 1.8 \\
\hline & I strongly disagree & 15 & 3.4 & 4 & 2.9 & 11 & 3.7 & 3 & 3.0 & 12 & 3.6 \\
\hline \multirow[t]{5}{*}{ Patient's autonomy } & I strongly agree & 153 & 34.9 & 66 & 48.2 & 87 & 28.9 & 36 & 35.6 & 115 & 34.6 \\
\hline & I agree & 136 & 31.1 & 41 & 29.9 & 95 & 31.6 & 30 & 29.7 & 104 & 31.3 \\
\hline & I cannot decide & 84 & 19.2 & 17 & 12.4 & 67 & 22.3 & 22 & 21.8 & 62 & 18.7 \\
\hline & I disagree & 18 & 4.1 & 5 & 3.7 & 13 & 4.3 & 7 & 6.9 & 11 & 3.3 \\
\hline & I strongly disagree & 18 & 4.1 & 4 & 2.9 & 14 & 4.7 & 3 & 3.0 & 15 & 4.5 \\
\hline \multirow[t]{5}{*}{ Treatment costs } & I strongly agree & 67 & 15.3 & 13 & 9.5 & 54 & 17.9 & 14 & 13.9 & 53 & 16.0 \\
\hline & I agree & 100 & 22.8 & 26 & 19.0 & 74 & 24.6 & 21 & 20.8 & 77 & 23.2 \\
\hline & I cannot decide & 91 & 20.8 & 35 & 25.6 & 56 & 18.6 & 29 & 28.7 & 62 & 18.7 \\
\hline & I disagree & 87 & 19.9 & 29 & 21.2 & 58 & 19.3 & 20 & 19.8 & 67 & 20.2 \\
\hline & I strongly disagree & 73 & 16.7 & 32 & 23.4 & 41 & 13.6 & 16 & 15.8 & 55 & 16.6 \\
\hline \multirow[t]{5}{*}{ ADs } & I strongly agree & 209 & 47.7 & 67 & 48.9 & 142 & 47.2 & 41 & 40.6 & 165 & 49.7 \\
\hline & I agree & 124 & 28.3 & 38 & 27.7 & 86 & 28.6 & 30 & 29.7 & 93 & 28.0 \\
\hline & I cannot decide & 61 & 13.9 & 18 & 13.1 & 43 & 14.3 & 20 & 19.8 & 40 & 12.1 \\
\hline & I disagree & 18 & 4.1 & 9 & 6.6 & 9 & 3.0 & 8 & 7.9 & 10 & 3.0 \\
\hline & I strongly disagree & 12 & 2.7 & 4 & 2.9 & 8 & 2.7 & 2 & 2.0 & 10 & 3.0 \\
\hline \multirow{5}{*}{$\begin{array}{l}\text { Wishes expressed by the } \\
\text { family/legal guardians }\end{array}$} & I strongly agree & 94 & 21.5 & 16 & 11.7 & 78 & 25.9 & 10 & 9.9 & 83 & 25.0 \\
\hline & I agree & 162 & 37.0 & 43 & 31.4 & 119 & 39.5 & 36 & 35.6 & 123 & 37.1 \\
\hline & I cannot decide & 107 & 24.4 & 43 & 31.4 & 64 & 21.3 & 35 & 34.7 & 71 & 21.4 \\
\hline & I disagree & 37 & 8.5 & 20 & 14.6 & 17 & 5.7 & 12 & 11.9 & 25 & 7.5 \\
\hline & I strongly disagree & 22 & 5.0 & 13 & 9.5 & 9 & 3.0 & 8 & 7.9 & 14 & 4.2 \\
\hline \multirow[t]{5}{*}{ Legal regulations } & I strongly agree & 159 & 36.3 & 64 & 46.7 & 95 & 31.6 & 34 & 33.7 & 124 & 37.4 \\
\hline & I agree & 140 & 32.0 & 39 & 28.5 & 101 & 33.6 & 32 & 31.7 & 106 & 31.9 \\
\hline & I cannot decide & 75 & 17.1 & 17 & 12.4 & 58 & 19.3 & 18 & 17.8 & 55 & 16.6 \\
\hline & I disagree & 29 & 6.6 & 8 & 5.8 & 21 & 7.0 & 11 & 10.9 & 18 & 5.4 \\
\hline & I strongly disagree & 15 & 3.4 & 6 & 4.4 & 9 & 3.0 & 4 & 4.0 & 11 & 3.3 \\
\hline \multirow[t]{5}{*}{ Religious principles } & I strongly agree & 82 & 18.7 & 30 & 21.9 & 52 & 17.3 & 18 & 17.8 & 63 & 19.0 \\
\hline & I agree & 138 & 31.5 & 44 & 32.1 & 94 & 31.2 & 27 & 26.7 & 110 & 33.1 \\
\hline & I cannot decide & 130 & 29.7 & 32 & 23.4 & 98 & 32.6 & 37 & 36.6 & 91 & 27.4 \\
\hline & I disagree & 36 & 8.2 & 17 & 12.4 & 19 & 6.3 & 10 & 9.9 & 26 & 7.8 \\
\hline & I strongly disagree & 34 & 7.8 & 13 & 9.5 & 21 & 7.0 & 8 & 7.9 & 26 & 7.8 \\
\hline \multirow[t]{5}{*}{ Need for beds in the ICU } & I strongly agree & 28 & 6.4 & 3 & 2.2 & 25 & 8.3 & 3 & 3.0 & 25 & 7.5 \\
\hline & I agree & 31 & 7.1 & 10 & 7.3 & 21 & 7.0 & 10 & 9.9 & 21 & 6.3 \\
\hline & I cannot decide & 65 & 14.8 & 10 & 7.3 & 55 & 18.3 & 14 & 13.9 & 50 & 15.1 \\
\hline & I disagree & 89 & 20.3 & 32 & 23.4 & 57 & 18.9 & 28 & 27.7 & 61 & 18.4 \\
\hline & I strongly disagree & 205 & 46.8 & 80 & 58.4 & 125 & 41.5 & 46 & 45.5 & 156 & 47.0 \\
\hline
\end{tabular}

ICU intensive care unit; $L S T$ life-sustaining treatment; $A D$ advance directives 
The sum of squared loadings, proportional and cumulative variance, shown in Table 5, provide more information on relevancy and the information provided by the factors. Due to the middling results of KMO, the factors have moderate contribution to the explained variance.

The exploratory factor analysis yielded 8 different factors. One factor has subsequently been reduced to one question. All of the questions in that factor were related to the topic of parties included in the decision-making process. However, due to the way the questions were formulated, it was not possible to analyse them as one factor. Therefore, we decided to focus on the question pertaining to the inclusion of nurses in the decision-making process.

Factor were analysed according to the hospital type, ICU type, age, sex, vocation, level of education, total work experience, ICU work experience and specialisation.

List of factors, cumulative variance explained by each factor, comprising questions and the sum of squared loading are shown in Table 6.

Analysis of the factors showed that physicians were more inclined to respect patient's wishes then nurses with high school education $(p=0.038)$, however nurses with high school $(p<0.001)$, college $(p=0.005)$ and university education $(p=0.003)$ were more inclined to respect religious and cultural principles than physicians.

Participants younger then 31 years are more inclined to respect religious and cultural principles than those aged $32-44(p=0.022)$.

A higher inclination towards paramedical aspects of decision-making process was noted in neurological and multidisciplinary ICUs compared to surgical $(p<0.001$ and $p=0.044$, respectively), neurologists compared with anaesthesiologists $(p=0.019)$, medical professionals aged 45-57 years in comparison to those aged less than 31 years $(p=0.003)$, male participants compared to female participants $(p=0.001)$, and physicians compared to nurses with high school $(p<0.001)$, college $(p<0.001)$ and university education $(p=0.014)$.
Analysis showed that nurses were more included in the decision-making process in neurological more than in surgical, medical, or multidisciplinary ICUs $(p<0.001$, $p=0.005, p=0.023$ respectively). They were also more included in surgical than in medical ICUs $(p=0.005)$.

Male participants and physicians were more prone to withholding of LST, instigating DNACPR orders and withdrawing of antibiotics and inotropes than female participants and nurses with college and university education $(p<0.001$ in all cases).

Withdrawal of mechanical ventilation, endotracheal tubes and hydration was more common in clinical compared to general hospitals $(p=0.016)$, and in neurological ICUs compared to surgical $(p=0.031)$, medical $(p=0.005)$, or multidisciplinary $(p=0.003)$.

Male participants in comparison to female $(p=0.002)$, physicians in comparison to nurses with high school and college education $(p<0.001$ in both cases), and medical professionals aged 32-57 years in comparison to those aged less than 31 years $(p<0.001)$ displayed more liberal attitudes about LST limitation.

No significant differences were noted among the groups regarding disagreement in the decision-making process.

\section{Discussion}

This is the first study to assess the experiences and attitudes of medical professionals working in ICUs in Croatia on the treatment of end-of-life patients. Our results show that LST limitations occur less frequently than in other countries, even though they were found ethically acceptable by most of the participants. This may be caused by the discrepancy between the attitudes created by the reality ICU medical professionals witness on a daily basis and what is allowed by the law. Croatia is a mainly catholic country [26] and paternalistic and conservative attitudes are expected considering geographical location in Southern Europe, as found by previous studies [3-7].

Table 5 Sum of squared loadings, proportional variance, and cumulative variance for each factor

\begin{tabular}{llcc}
\hline Factor & Sum of squared loadings & Proportional variance (\%) & $\begin{array}{c}\text { Cumulative } \\
\text { variance (\%) }\end{array}$ \\
\hline Respecting patients' wishes & & 13.9 & 13.9 \\
Respecting religious and cultural principles & 2.5033 & 8.5 & 22.4 \\
Paramedical aspects of decision-making & 1.5305 & 8.1 & 30.5 \\
Decision-making process including nurses & 1.4549 & 5.5 & 36 \\
Common withdrawal of therapies & 0.9838 & 12.4 & 8.9 \\
Uncommon withdrawal of therapies & 2.1067 & 7.4 & 21.3 \\
Disagreement in decision-making & 1.5147 & 6.2 & 28.7 \\
Liberal attitudes towards LST limitation & 1.2628 & 35 \\
\hline
\end{tabular}


Table 6 List of factors, cumulative variance, comprising questions and the sum of squared loadings

\begin{tabular}{|c|c|c|}
\hline $\begin{array}{l}\text { Factor name (cumulative variance explained by } \\
\text { each factor) }\end{array}$ & Comprising questions & $\begin{array}{l}\text { Sum of } \\
\text { squared } \\
\text { loadings }\end{array}$ \\
\hline \multirow[t]{7}{*}{ Respecting patients' wishes (13.9\%) } & Patients'interests should be respected in LST limitation decision-making & 0.8173 \\
\hline & Patients'autonomy should be respected in LST limitation decision-making & 0.6914 \\
\hline & AD should be respected in LST limitation decision-making & 0.6039 \\
\hline & Good medical practice should be respected in LST limitation decision-making & 0.5534 \\
\hline & Legal regulations should be respected in LST limitation decision-making & 0.5242 \\
\hline & Families' wishes should be respected in LST limitation decision-making & 0.4182 \\
\hline & How often are you acquainted with patients' and families' wishes? & 0.1061 \\
\hline \multirow[t]{4}{*}{ Respecting religious and cultural principles (22.4\%) } & $\begin{array}{l}\text { Religious and cultural principles expressed by the patient or family should be } \\
\text { respected }\end{array}$ & 1.0298 \\
\hline & Religious principles should be respected in LST limitation decision-making & 0.4488 \\
\hline & Do you think AD are helpful in the decision-making process? & 0.3148 \\
\hline & Religious and cultural principles expressed by the physician should be respected & 0.2408 \\
\hline \multirow[t]{3}{*}{ Paramedical aspects of decision-making (30.5\%) } & Need for beds in the ICU should be respected in LST limitation decision-making & 0.7197 \\
\hline & Treatment costs should be respected in LST limitation decision-making & 0.6231 \\
\hline & Is health care resource allocation important in decision-making? & 0.5253 \\
\hline Decision-making process including nurses (36\%) & LST limitation decision-making process includes ICU physicians and nurses & 0.6929 \\
\hline \multirow[t]{4}{*}{ Common withdrawal of therapies (12.4\%) } & Are decisions to withdraw antibiotics made in your ICU? & 0.7639 \\
\hline & Are decisions to withdraw inotropes made in your ICU? & 0.7477 \\
\hline & Are decisions to withhold LST made in your ICU? & 0.7069 \\
\hline & Are DNACPR decisions made in your ICU? & 0.6075 \\
\hline \multirow[t]{4}{*}{ Uncommon withdrawal of therapies (21.3\%) } & Are decisions to withdraw endotracheal tube made in your ICU? & 0.876 \\
\hline & Are decisions to withdraw mechanical ventilation made in your ICU? & 0.6829 \\
\hline & Are decisions to withdraw hydration made in your ICU? & 0.4667 \\
\hline & Do you agree that hydration should be withdrawn in end-of-life patients? & 0.0654 \\
\hline \multirow[t]{5}{*}{ Disagreement in decision-making (28.7\%) } & How often is agreement between physicians not achieved? & 0.7366 \\
\hline & $\begin{array}{l}\text { How often is agreement between physicians and family/legal guardians not } \\
\text { achieved? }\end{array}$ & 0.6122 \\
\hline & Have you ever disagreed with the method of LST limitation? & 0.5269 \\
\hline & $\begin{array}{l}\text { Have you ever refused to be a part of decision-making discussion or implementa- } \\
\text { tion? }\end{array}$ & 0.1134 \\
\hline & $\begin{array}{l}\text { Do you think there is a difference between withholding and withdrawing LST from } \\
\text { an ethical standpoint? }\end{array}$ & 0.0628 \\
\hline \multirow[t]{4}{*}{ Liberal attitudes towards LST limitation (35\%) } & $\begin{array}{l}\text { Do you think that withholding and withdrawing LST in end-of-life patients is ethi- } \\
\text { cally acceptable? }\end{array}$ & 0.694 \\
\hline & Do you think DNACPR decisions in end-of-life patients are ethically acceptable? & 0.5893 \\
\hline & Do you respect DNACPR decisions? & 0.2799 \\
\hline & $\begin{array}{l}\text { Do you think LST limitation is the same from an ethical standpoint in the adult } \\
\text { patients who are brain dead, terminally ill or in a vegetative state? }\end{array}$ & 0.1232 \\
\hline
\end{tabular}

ICU intensive care unit; LST life-sustaining treatment; DNACPR do-not-attempt cardiopulmonary resuscitation

American Society of Critical Care Medicine has stated back in 1989 that LST limitations are ethically appropriate in certain cases [27]. More recent research conducted in the Netherlands, Switzerland, Denmark, Sweden, Belgium and Italy showed that $23-51 \%$ of patients died after a decision to limit LST has been made [28], while Ethicus-2 study showed that such a decision is made in as much as $12 \%$ of patients admitted to ICU and in almost $81 \%$ of the study population, which included patients who died in the ICU. It also showed that withholding of LST occurred in $44 \%$ and withdrawing of LST occurred in $36 \%$ of the study population [7]. A study conducted in the ICUs in the city of Milan, Italy, showed that $73 \%$ of physicians indicated that DNACPR orders were used in their ICU [29].

Our research shows that LST limitation does not occur often, as only $18 \%$ of participants stated that DNACPR orders were frequently made in their ICUs, and only 
$13 \%$ of participants stated that therapy was frequently withheld. Study of experiences in Slovene ICUs showed a DNACPR orders are made more commonly than decisions to withhold treatments [25]. However, $67 \%$ of Slovene physicians frequently make DNACPR decisions, as opposed to $38 \%$ of Croatian physicians.

Studies conducted in Germany, Italy and Denmark also showed that DNACPR orders are made often and are more frequent than limitation of antibiotics and vasoactive medications [29-31]. The results of a multicentric study conducted in Spain are consistent with previous studies which showed that, in comparison to Northern European countries, DNACPR decisions were less frequently noted in the patient's medical documents and less LST limitation decision were made [32].

Even though withdrawal of mechanical ventilation, endotracheal tubes and hydration is not very common in Croatian ICUs, it is more common in clinical compared to general hospitals. Research conducted by Bach showed that university-based intensivists were more prone to instigating DNACPR orders and withdrawing LST than community-based intensivists [33].

Most participants in our study found that DNACPR orders and withholding/withdrawing of LST were ethically acceptable, and DNACPR orders were always respected by $67.4 \%$ of participants. Thirty seven percent of participants stated that there was a difference between withholding and withdrawing LST from an ethical standpoint. Many end-of-life guidelines purport that there is no ethical difference between withholding and withdrawing of LST, which is supported by ethical principles of professional duty, beneficence, nonmaleficence and autonomy [15].

Nevertheless, almost half of participants in a study conducted in Milanese ICUs stated that there is a difference [29]. Studies exploring nurses' attitudes also found that about half of nurses find that withholding of LST is not morally the same as withdrawal $[22,34,35]$. Seventy percent of participants in a study conducted in tertiary care hospitals in Sri Lanka responded they found withholding LST more comfortable then withdrawing it [36].

Involvement of nurses in end-of-life decision-making process is a widely accepted attitude. Nonetheless, multiple studies confirm that nurses are not sufficiently included. Our results show that only $28 \%$ of physicians and $21 \%$ of nurses stated that nurses were included in the decision-making, while almost $50 \%$ of physicians stated they did not include nurses. Around $60 \%$ of Slovene intensivists stated they never included nurses in such decisions, and only $5 \%$ stated they were always included [25]. Half of participants in a study conducted in Germany [30] and 90\% of participants in Portugal [37] stated that nurses were not included in the decision-making.
Similar results were found in studies conducted in Italy and Hong Kong [29, 38]. Studies exploring nurses' attitudes and experiences on the matter found that nurses thought they were not included, and their opinions were not esteemed [18-21, 39].

A study conducted in France in 2003. showed that, despite the opinion that nurses should be included in the decision-making process, $50 \%$ of physicians and only $27 \%$ of nurses stated it occurred in practice [40]. Another study conducted in France after a law allowing withholding and withdrawing of LST was passed, showed an improvement [41]. This is an encouraging example of how a change of legal aspects can positively affect everyday practice.

Apart from not being sufficiently included in the decision-making process, nurses are not adequately active in initiating discussions about LST limitation. Our research showed that only $2 \%$ of physicians and $3 \%$ of nurses stated it were nurses who initiated such discussions. This is confirmed by other studies with similar findings [19, $34,42]$. Badir suggests the fact that nurses fail to initiate LST limitation discussions is a source of ethical concern, as in ensuring quality end-of-life care it is important that nurses learn and meet the needs and expectations of patients who seek a dignified death [22].

Analysis of the factors in our study showed that physicians were more inclined to respect patient's wishes then nurses with high school education. Other research showed that more experienced physicians were more inclined to take patient's wishes in account in end-of-life decision-making [29], and that more male than female physicians found patient's wishes to be the most important criterion in LST limitation decision-making [37]. Our research did not find such differences.

Nevertheless, Croatian ICU nurses of all levels of education were more inclined to respect religious and cultural principles than physicians. A study from South Africa points to the same direction, as $75 \%$ and $63 \%$ of nurses declared that patient's and families' religious beliefs, respectively, are important in the decision-making process [34].

Our study shows that most of the participants found patient's interests and autonomy to be an important aspect to be considered when making end-of-life decisions. Most of them also stated that a verbal or written decision made by a capable patient should be respected. However, $55.2 \%$ of participants stated that they rarely knew the patient's wishes regarding LST limitation. Therefore, a conclusion can be extracted that Croatian medical professionals find autonomy to be an important principle, but they are not adequately informed about patient's wishes, which casts a doubt on whether those wishes are actually respected. Ethical principles of 
autonomy, privacy and nonmaleficence underpin the significance and importance of respecting patient's wishes. End-of-life guidelines affirm the pertinence of encouraging patients to express their will and wishes while capable for it to be respected once they become incompetent [15]. Medical professionals should motivate patients to express their opinions and wishes [43].

Seventy six percent of participants in our research stated that AD should be respected, but it is almost never encountered in their practice. A study conducted in Slovene ICUs also found that physicians rarely encountered $\mathrm{AD}$ [25], and a study from Milan showed that $70 \%$ of physicians were not acquainted with the notion of $\mathrm{AD}$ [29].

This study has several limitations. The total response rate was not as high as expected and there is a possibility of bias, as it may be that most of the participants have a special interest in the topic and were more inclined to fill out the questionnaire. The research was not conducted in all the hospitals in the Republic of Croatia even though it did cover all geographic regions, and residents were not included. All steps were taken to protect participant anonymity, however, since certain actions described in the questionnaire are not allowed according to Croatian law, it is possible that some participants adjusted their responses.

\section{Conclusion}

Our study has found that DNACPR orders are not commonly made in Croatian ICUs, even though limitations of LST were found ethically acceptable by most of the participants. It has also shown the inadequate involvement of nurses in the decision-making process. The results have confirmed our expectations of paternalistic and conservative attitudes considering Croatia's geographical location in Southern Europe.

This was the first study about medical professionals' attitudes and experiences on treatment of end-of-life patients in ICUs in Croatia and has provided an insight into the current state of the issue. In addition, it confirms the findings of previous studies, and it can be used to help evaluate and compare the situation in other neighbouring countries which are in a similar socio-economic situation.

This type of research should be repeated in the future to assess possible changes, and to provide more data which would help in making and shaping the guidelines and legally binding policies on treatment of end-of-life patients in Croatia.

\section{Abbreviations}

ICU: Intensive care unit; LST: Life-sustaining treatment; DNACPR: Do-notattempt cardiopulmonary resuscitation; AD: Advance directives.

\section{Supplementary Information}

The online version contains supplementary material available at https://doi. org/10.1186/s12910-022-00752-5.

Additional file 1. The Questionnaire. The questionnaire used in this research, translated in English.

\section{Acknowledgements}

None.

\section{Authors' contributions}

DS, SG, DT, MKT, AS and AB made substantial contributions to the conception and design of the study. JP, VM, TK, RR, ZP, VNA, MV, NK, KC, ZZ, IG, JK, SV, $\mathrm{RK}, \mathrm{BN}$ and $M G$ were involved in the acquisition and interpretation of data. AS and MKT did the statistical analysis. DS wrote the article. AB, AS and MKT substantially revised the work. All authors critically appraised the paper for its intellectual content, read and approved the manuscript.

\section{Funding}

This article is a part of 'Values and decisions at the end of life' (VAL-DEEND) project funded by Croatian Science Foundation (Grant Number: IP-2016-06-2721).

\section{Availability of data and materials}

All data generated or analysed during this study are included in this published article [and its Additional file 1].

\section{Declarations}

\section{Ethics approval and consent to participate}

An information sheet of the study was attached to the questionnaire that was provided to each of the participants. Completing and returning of the questionnaire was taken as implied informed consent to participate in the study. Ethical approval was obtained from the ethics committees of all the institutions involved in the research (University Hospital Centre Zagreb 2/21AG 16.5.2017; Clinical Hospital Centre Rijeka 2170-29-02/15-17-2 23.5.2017; University Hospital Center Split 2181-147-01/06/M.S.-17-2, 20.4.2017; Sestre Milosrdnice Clinical Hospital Center EP-7259/17-13, 5.5.2017; Clinical Hospital Sveti Duh 01-1914, 1.6.207; General Hospital 'Dr. Josip Benčević'Slavonski Brod 43800/18-2303, 26.09.2018; General Hospital Šibenik 01-16852/1-18, 03.10.2018; General Hospital Varaždin02/1-91/89-2018, 23.10.2018; University Hospital Dubrava 17.5.2017. no number provided), as well as from the ethics committee of the School of Medicine, University of Zagreb (380-59-10106-17$100 / 208,13.7 .2017)$. All methods of research were performed in accordance with the Declaration of Helsinki.

\section{Consent for publication}

Not applicable.

\section{Competing interests}

The authors declare that they have no competing interests.

\section{Author details}

${ }^{1}$ University Hospital Dubrava, Av. Gojka Šuška 6, 10000 Zagreb, Croatia. ${ }^{2}$ Sestre Milosrdnice University Hospital Centre, Vinogradska cesta 29, 10000 Zagreb, Croatia. ${ }^{3}$ University Hospital Centre Zagreb, Kišpatićeva ul. 12, 10000 Zagreb, Croatia. ${ }^{4}$ Clinical Hospital Sveti Duh, UI. Sveti Duh 64, 10000 Zagreb, Croatia. ${ }^{5}$ University Hospital Centre Split, Spinčićeva 1, 21000 Split, Croatia. ${ }^{6}$ Clinical Hospital Centre Rijeka, Krešimirova ul. 42, 51000 Rijeka, Croatia. ${ }^{7}$ General Hospital 'Dr. Josip Benčević' Slavonski Brod, UI. Andrije Štampara, 35000 Slavonski Brod, Croatia. ${ }^{8}$ General Hospital Šibenik, Ul. Stjepana Radića 83, 22000 Šibenik, Croatia. ${ }^{9}$ General Hospital Varaždin, Ul. Ivana Meštrovića 1, 42000 Varaždin, Croatia. ${ }^{10}$ Marien Hospital Dusseldorf, Rochusstraße 2, 40479 Düsseldorf, Germany. ${ }^{11}$ University Medical Centre Ljubljana, Bohoričeva 20, 1000 Ljubljana, Slovenia. ${ }^{12}$ Andrija Štampar School of Public Health, School of Medicine, University of Zagreb, Johna Davidsona Rockfellera 4, 10000 Zagreb, Croatia. 
Received: 8 December 2021 Accepted: 8 February 2022

Published online: 16 February 2022

\section{References}

1. Thelen M. End-of-life decision making in intensive care. Crit Care Nurse. 2005;25(6):28-37.

2. Piers RD, Azoulay E, Ricou B, Dekeyser Ganz F, Decruyenaere J, Max A, et al. Perceptions of appropriateness of care among European and Israeli intensive care unit nurses and physicians. JAMA. 2011;306(24):2694-703.

3. Sprung CL, Cohen SL, Sjokvist P, Baras M, Bulow HH, Hovilehto S, i sur. Endof-life practices in European Intensive Care Units: the Ethicus study. JAMA. 2003;290(6):790-7.

4. Vincent JL. Forgoing life support in western European intensive care units: the results of an ethical questionnaire. Crit Care Med. 1999;27(8):1626-33.

5. Vincent $J$ L. European attitudes towards ethical problems in intensive care medicine: results of an ethical questionnaire. Intensive Care Med. 1990;16(4):256-64.

6. Bülow HH, Sprung CL, Baras M, Carmel S, Svantesson M, Benbenishty J, et al. Are religion and religiosity important to end-of-life decisions and patient autonomy in the ICU? The Ethicatt study. Intensive Care Med. 2012;38(7):1126-33.

7. Avidan A, Sprung CL, Schefold JC, Ricou B, Hartog CS, Nates JL, et al. Variations in end-of-life practices in intensive care units worldwide (Ethicus-2): a prospective observational study. Lancet Respir Med. 2021;9(10):1101-10.

8. SIAARTI_Italian Society of Anesthesia, Analgesia, Resuscitation and Intensive Care Bioethical Board. End-of-life care and the intensivist: SIAARTI recommendations on the management of the dying patient. Minerva Anestesiol. 2006;72:927-63.

9. Skupna izjava Slovenskega združenja za intenzivno medicino in Komisije RS za medicinsko etiko: Etična priporočila za odločanje o zdravljenju in paliativni oskbri bolnika ob koncu življenja v intenzivni medicini. Ljubljana; 2015.

10. Downar J, Delaney JW, Hawryluck L, Kenny L. Guidelines for the withdrawal of life-sustaining measures. Intensive Care Med. 2016:42:1002-17.

11. Truog RD, Campbell ML, Curtis JR, Haas CE, Luce JM, Rubenfeld GD, et al. Recommendations for end-of-life care in the intensive care unit: a consensus statement by the American College of Critical Care Medicine. Crit Care Med. 2008;36(3):953-63.

12. Valentin A, Druml W, Steltzer H, Wiedermann CJ. Recommendations on therapy limitation and therapy discontinuation in intensive care units: consensus paper of the Austrian associations of intensive care medicine. Intensive Care Med. 2008;34(4):771-6.

13. Myatra SN, Salins N, Iyer S, Macaden SC, Divatia JV, Muckaden M, et al. Endof-life care policy: an integrated care plan for the dying. Indian J Crit Care Med. 2014;18(9):615-35.

14. Bandrauk N, Downar J, Paunovic B. Withholding and withdrawing lifesustaining treatment: the Canadian critical care society position paper. Can J Anaesth. 2018;65:105-22.

15. Spoljar D, Curkovic M, Gastmans C, Gordijn B, Vrkic D, Jozepovic A, et al. Ethical content of expert recommendations for end-of-life decision-making in intensive care units: a systematic review. J Crit Care. 2020;58:10-9.

16. Curtis JR, Vincent JL. Ethics and end-of-life care for adults in the intensive care unit. Lancet. 2010;376:1347-53.

17. Bach V, Ploeg J, Black M. Nursing roles in end-of-life decision making in critical care settings. West J Nurs Res. 2009;31:496-512.

18. Flannery L, Peters K, Ramjan LM. The differing perspectives of doctors and nurses in end-of-life decisions in the intensive care unit: a qualitative study. Aust Crit Care. 2020;33(4):311-6.

19. Benbenishty J, Ganz FD, Lippert A, Bulow H-H, Wennberg E, Henderson B, et al. Nurse involvement in end-of-life decision making: the ETHICUS study. Intensive Care Med. 2006:32(1):129-32.

20. Griffiths I. What are the challenges for nurses when providing end-of-life care in intensive care units? Br J Nurs. 2019;28(16):1047-52.

21. Velarde-García JF, Luengo-González R, González-Hervías R, Cardenete-Reyes C, Álvarez-Embarba B, Palacios-Ceña D. Limitation of therapeutic effort experienced by intensive care nurses. Nurs Ethics. 2018;25(7):867-79.

22. Badır A, Topçu I, Türkmen E, Göktepe N, Miral M, Ersoy N, et al. Turkish critical care nurses' views on end-of-life decision making and practices. Nurs Crit Care. 2016:21(6):334-42
23. Zakon o zdravstvenoj zaštiti. https://www.zakon.hr/z/190/Zakon-o-zdrav stvenoj-zaštiti. Accessed 31.10.2021.

24. Zakon o zaštiti prava pacijenata. https://www.zakon.hr/z/255/Zakon-ozaštiti-prava-pacijenata. Accessed 31.10.2021.

25. Groselj U, Orazem M, Kanic M, Vidmar G, Grosek S. Experiences of Slovene ICU physicians with end-of-life decision making: a nation-wide survey. Med Sci Monit. 2014;20:2007-12.

26. Popis stanovništva, kućanstava i stanova 2011. https://www.dzs.hr/hrv/ censuses/census2011/results/htm/H01_01_12/H01_01_12.html. Accessed 31.10.2021.

27. Task Force on Ethics of the Society of Critical Care Medicine. Consensus report on the ethics of foregoing life-sustaining treatments in the critically ill. Crit Care Med. 1990;18:1435-9.

28. van der Heide A, Deliens L, Faisst K, Nilstun T, Norup M, Paci E, et al. EURELD consortium. End-of-life decision-making in six European countries: descriptive study. Lancet. 2003:362(9381):345-50.

29. Giannini A, Pessina A, Tacchi EM. End-of-life decisions in intensive care units: attitudes of physicians in an Italian urban setting. Intensive Care Med. 2003;29(11):1902-10.

30. Jox RJ, Krebs M, Fegg M, Reiter-Theil S, Frey L, Eisenmenger W, et al. Limiting life-sustaining treatment in German intensive care units: a multiprofessional survey. J Crit Care. 2010;25(3):413-9.

31. Christensen L, Jensen H, Kristensen S, Goldinger M, Gjedsted J, Christensen S, et al. Treatment limitations in intensive care units. Dan Med J. 2021;68(8):A03210235.

32. Esteban A, Gordo F, Solsona JF, Alía I, Caballero J, Bouza C, et al. Withdrawing and withholding life support in the intensive care unit: a Spanish prospective multi-centre observational study. Intensive Care Med. 2001:27(11):1744-9.

33. Bach PB, Carson SS, Leff A. Outcomes and resource utilization for patients with prolonged critical illness managed by university-based or communitybased subspecialists. Am J Respir Crit Care Med. 1998;158(5):1410-5.

34. Langley G, Schmollgruber S, Fulbrook P, Albarran JW, Latour JM. South African critical care nurses'views on end-of-life decision-making and practices: South African critical care nurses' views on EOL decision-making and practices. Nurs Crit Care. 2014;19(1):9-17.

35. Latour JM, Fulbrook P, Albarran JW. EfCCNa survey: European intensive care nurses' attitudes and beliefs towards end-of-life care. Nurs Crit Care. 2009;14(3):110-21.

36. Chang T, Darshani S, Manikavasagam P, Arambepola C. Knowledge and attitudes about end-of-life decisions, good death and principles of medical ethics among doctors in tertiary care hospitals in Sri Lanka: a cross-sectional study. BMC Med Ethics. 2021;22(1):66.

37. Cardoso T, Fonseca T, Pereira S, Lencastre L. Life-sustaining treatment decisions in Portuguese intensive care units: a national survey of intensive care physicians. Crit Care. 2003;7(6):R167-75.

38. Yap H, Joynt GM, Gomersall CD. Ethical attitudes of intensive care physicians in Hong Kong: questionnaire survey. Hong Kong Med J. 2004;10(4):244-50.

39. Flannery L, Ramjan LM, Peters K. End-of-life decisions in the Intensive Care Unit (ICU) - exploring the experiences of ICU nurses and doctors - a critical literature review. Aust Crit Care. 2016;29(2):97-103.

40. Ferrand E, Lemaire F, Regnier B, Kuteifan K, Badet M, Asfar P, et al. Discrepancies between perceptions by physicians and nursing staff of intensive care unit end-of-life decisions. Am J Respir Crit Care Med. 2003;167(10):1310-5.

41. Blythe JA, Kentish-Barnes N, Debue AS, Dohan D, Azoulay E, Covinsky $\mathrm{K}$, et al. An interprofessional process for the limitation of life-sustaining treatments at the end of life in France. J Pain Symptom Manag. 2021;:S0885-3924(21):00399-407.

42. Ho KM, English S, Bell J. The involvement of intensive care nurses in end-oflife decisions: a nationwide survey. Intensive Care Med. 2005;31(5):668-73.

43. Gruppo di Studio ad Hoc della Commissione di Bioetica della SIAARTI. SIAARTI guidelines for admission to and discharge from intensive care units and for the limitation of treatment in intensive care. Minerva Anestesiol. 2003;69:101-18.

\section{Publisher's Note}

Springer Nature remains neutral with regard to jurisdictional claims in published maps and institutional affiliations. 